\title{
Dickkopf-1 Expression as a Predicting Factor for Bone Metastasis in Breast Cancer
}

\author{
Faruly Wijaya S. LIMBA ${ }^{1}$, Erwin Danil YULIAN ${ }^{1}$, Diani KARTINI ${ }^{1}$, Bob ANDINATA ${ }^{2}$, \\ Hartono TJAHJADI ${ }^{3}$, Dhanasari VIDIAWATI ${ }^{4}$ \\ ${ }^{1}$ Universitas Indonesia, Faculty of Medicine, Dr. Cipto Mangunkusumo General Hospital, \\ Department of Surgery, Division of Surgical Oncology \\ ${ }^{2}$ National Cancer Center, Dharmais Hospital, Department of Surgical Oncology \\ ${ }^{3}$ Universitas Indonesia, Faculty of Medicine, Dr. Cipto Mangunkusumo General Hospital, \\ Department of Pathological Anatomy \\ ${ }^{4}$ Universitas Indonesia, Faculty of Medicine, Department of Community Medicine, Jakarta, INDONESIA
}

\begin{abstract}
Bone is the main metastatic site in breast cancer patients. A marker is needed for early identification of breast cancer patients who are at risk of bone metastasis. Dickkopf-1 (DKK-1) plays an early role in the cascade of bone metastasis process in breast cancer. Therefore, detection of its expression is presumed to predict bone metastasis better than other biomarkers. This study was aimed to determine the potential of DKK-1 expression as a predictor of bone metastasis in breast cancer and its association with clinicopathological factors. The study design was a retrospective cohort study of medical records in the Surgical Oncology Division, Department of Surgery, Cipto Mangunkusumo General Hospital, Jakarta, Indonesia from October 2018 to June 2019. Bivariate analysis was performed to analyse data using Chi square test or Fischer's exact test. We included 76 subjects divided into two groups: bone metastasis group $(n=38)$ and non-bone metastasis group $(n=38)$. The $H$-Score cut-off value for DKK-1 expression was 142.5. We found a statistically significant association between high expression of DKK-1 and the incidence of bone metastasis in breast cancer (odd ratio $[O R]=12.083,95 \%$ confidence interval $[C l]=4.101-35.600, p<0.001)$. Associations between DKK-1 expression and clinicopathological factors were not statistically significant. DKK-1 expression is potential to predict and an independent factor of the bone metastasis occurrence in breast cancer.
\end{abstract}

Keywords: Breast cancer, Bone metastatis, Dickkopf-1

\section{INTRODUCTION}

Breast cancer is the most frequent cancer among women in the world. Breast cancer accounts for $23 \%$ of all cancer cases and $14 \%$ of cancer deaths. ${ }^{1}$ In 2012, breast cancer in women was diagnosed in 1.7 million people, with an average of 43 per 100.000 population. $^{2}$ The case distribution is as follows: $46 \%$ in China, $14 \%$ in Japan, and $12 \%$ in Indonesia. ${ }^{2}$ In Indonesia, breast cancer is the most prevalent cancer with a prevalence of $18.6 \%$, an estimated incidence of 12 per 100.000 women, and mortality rate of 27 per $100.000 .^{3}$
Bone is the main site of metastasis in breast cancer patients; $75 \%$ of patients with stage IV breast cancer experience bone metastasis. ${ }^{4-6}$ Large cohort studies involving 7064 patients with early-stage breast cancer found that $22 \%$ of patients had bone metastasis at the average follow-up period of 8.4 years. ${ }^{7}$ The median overall survival (OS) of breast cancer patients with bone metastasis is 40 months. ${ }^{4}$ However, rapid diagnosis of bone metastasis is often hampered by slow radiological changes associated with disease progression. The radiological appearance of bone metastasis can change with time, both spontaneously and because of the effects of anti-resorption drugs. ${ }^{8}$ 
In addition, bone metastasis have similar characteristics to many non-malignant bone disease, making diagnosis more complicated. For this reason, a marker is needed for early identification of patients who are at high risk of bone metastasis, so that therapeutic strategies for this population can be optimized.

Various biomarkers have been found to be associated with breast cancer cells' tendency to metastasize to bones; highest associations are found in CXCR4 and RANK. ${ }^{6-7}$

The formation of breast cancer osteolytic lesions is caused by osteoclast stimulation and osteoblast inhibition. ${ }^{9}$ Osteoclast differentiation is regulated by osteoblasts. ${ }^{9}$ Osteoblasts produce osteoprotegrin (OPG), NF-kappa B (RANK) activator receptors and its ligands (RANKL). RANKL binds to RANK and increases osteoclast differentiation / activity (hematopoietic cell surface). In the skeletal system, DKK-1 is found in mature osteoblasts and osteocytes. DKK-1 regulates osteoblast differentiation. ${ }^{10}$ Mature osteoblasts increase regulation of OPG, which blocks RANK-L-induced osteoclastogenesis, causing inhibition of bone resorption. However, DKK-1 inhibits the formation and differentiation of osteoblasts by transferring the progenitors to adipogenesis. Preosteoblast increases bone resorption by increasing RANK-L which induces osteoclastogenesis. ${ }^{11}$

DKK-1 is known as a negative regulator of the Wnt signal pathway. ${ }^{12}$ DKK-1 binds to lipoprotein receptor-related protein-5/6 (LRP5/6) and inhibits interaction with Wnt- 1 , which results in $\beta$-catenin degradation and proliferation inhibition. ${ }^{13-15}$ Thus, there will be a decrease in the OPG: RANKL ratio, thereby increasing osteoclastogenesis and triggering osteoclastic bone resorption. Hence, when compared with CXCR4 and RANK, the DKK-1 upstream plays an earlier role in the cascade of bone metastasis processes. Therefore, detection of its expression is expected to be a better predicting factor.

Expressions and roles of DKK-1 differ in various cancers. Current studies have reported that excessive expression of DKK-1 is found in many malignant tumors, including lung cancer, esophageal carcinoma, cervical cancer, and hepatocellular car- cinoma, which shows the presence of oncogenic function of DKK-1. ${ }^{16-19}$ Notably, down-regulation is observed on the expression of DKK-1 in colon cancer, gastric cancer and melanoma. ${ }^{20-22}$

To date, there have been no studies on the expression of DKK-1 as a predictor of bone metastatic breast cancer in Indonesia. This study aimed to determine the potential of DKK-1 expression as a predictor of bone metastasis in breast cancer, and to explore the association between DKK-1 expression in metastatic breast cancer and clinicopathological factors.

\section{MATERIALS AND METHODS}

\section{Study Design and Subjects}

The study design was a retrospective cohort study. Data was obtained from patient medical records in the Surgical Oncology Division of the Department of Surgery, Cipto Mangunkusumo General Hospital (CMGH), Jakarta, Indonesia, from October 2018 to June 2019. There was no follow up on subjects in this study. The inclusion criteria of this study were breast cancer patients who had undergone mastectomy surgery at $\mathrm{CMGH}$ and were still in the post-discharge follow up sessions during the period of this study. This study was approved by the Ethics Committee of the Faculty of Medicine, Universitas Indonesia (approval number 1271/UN2.F1/ETIK/2018). The minimum number of subjects in this study was 76 subjects. This size was resulted from a calculation for test of difference in 2 independent proportions. The first proportion, stage 3 breast cancer patients with positive DKK-1, was 0.37 and the second proportion, lower than stage 3 breast cancer patients with negative DKK-1 expression, was $0.12 .{ }^{23}$

Subjects were categorized into 2 groups: bone metastasis group and non-bone metastasis group. Inclusion criteria of subjects are breast cancer patients who had undergone mastectomies in $\mathrm{CMGH}$, were still in the follow up period, and whose paraffin blocks were taken directly in the mastectomy surgeries. We excluded patients with incomplete medical records and patients whose paraffin block specimens were unavailable or unsuitable for immunohistochemical staining. We obtain demo- 
graphic, clinical, and pathological data of each patient from patient's medical records and pathology records. Breast cancer diagnosis was confirmed based on the pathological anatomy result. The age of the patient was resulted from the date of data collection subtracted by the date of birth. The stadium of cancer was considered from the clinical, ultrasonography, mammography, and postoperative examinations. Early stadium consisted of the first and second stadiums, meanwhile late local stadium consisted of the third stadium. The pathological grade had been evaluated by pathologists based on the Bloom-Richardson grading system. Menopause status of a subject was determined from the anamnesis data. The estrogen receptor, progesterone receptor, HER-2, KI-67, and molecular subtype data was collected from the IHC results. The bone metastasis status was determined from the bone scan and/or the spot fluoroscopy examination data.

\section{Immunohistochemistry (IHC) Staining of DKK-1}

Paraffin embedded tissue block was cut into $4 \mu \mathrm{m}$ each, placed on an object glass, and heated at $60^{\circ} \mathrm{C}$ in 60 minutes. Each section was deparaffinized using xylene for 3 times each for 3 minutes. Then each section was rehydrated through ethanol serial dilutions $(100 \%, 96 \%$, and $70 \%)$ each for 3 minutes and cleansed with water in 3 minutes. Endogen peroxidase blocking was conducted using $0.5 \% \mathrm{H}_{2} \mathrm{O}_{2}$ in methanol for 30 minutes, followed by water cleansing in 5 minutes. Pretreatment was carried out through antigen retrieval procedure in a $96^{\circ} \mathrm{C}$ decloaking chamber with $\mathrm{pH} 9.0$ Tris-EDTA (TE) in 10 minutes. Then, 10 minutes of peroxidase block was done. Three minutes of washing with $\mathrm{pH}$ 7.4 Phosphate-buffer saline (PBS) was conducted before and after the peroxidase block. Blocking background sniper was done in 10 minutes, then it washed with PBS in 3 minutes. Primary antibody against DKK-1 incubation in normal serum at a dilution of 1:50 was done in 60 minutes. DKK-1 mouse monoclonal antibody (B-7) from Santa Cruz Biotechnology was used in this study. Then the following steps were: 3 minutes of PBS washing, 30 minutes of post primary, 3 minutes of PBS washing, using polymer in 30 minutes, 5 minutes of PBS washing, using 3,3'-Diaminobenzidine (DAB) in 1-2 minutes, and water cleansing in 2 minutes. Ten seconds of counterstain using CAT hematoxylin was conducted. The sample was soaked in lithium carbonate (5\% in aquadest) in 5 seconds to make the nucleus into blue colored. Each section was dehydrated in ascending concentrations of ethanol for 3 minutes each, followed by clearance using xylene and cover slipped.

\section{IHC Staining Assessment}

Expression of DKK-1 was assessed by looking at staining intensity of the tumor cells' cytoplasm and counting the percentage of positive tumor cells. For each sample, 2-3 high power fields (400x) were randomly selected to analyze minimally 300 stained cells. Staining intensity of stained cells were counted using Image $\mathbf{J}$ (National Institutes of Health, USA). The level of staining intensity, compared to the positive controls, was categorized as the following: 0 (negative), +1 (weak positive),+ 2 (moderate positive), +3 (strong positive). ${ }^{24}$ The histology score (H-score) was calculated with the formula: $\mathrm{H}-\mathrm{Score}=(1 \mathrm{x}($ cell $\%+1)+(2 \times(\%$ cell $+2)+(3 \times(\%$ cell +3$)$. Then, the cut off values of DKK-1 expression were determined. DKK-1 expression was considered low if the $\mathrm{H}$-score $<$ cut off and high if the $\mathrm{H}$-score $\geq$ cut off.

\section{Statistical Analysis}

Data analysis was carried out using the IBM SPSS version 20. Bivariate analysis was performed using the Chi-square or Fisher's exact test. Moreover, multivariate analysis on clinicopathological factors used a logistic regression test. p-values of less than 0.05 were considered statistically significant.

\section{RESULTS}

\section{Characteristics of Patients}

There were 76 subjects in our study: 38 subjects in the bone metastasis group and 38 subjects in the non-bone metastasis group, all of which are included in the data analysis. Based on the bivariate analysis, HER-2 ( $\mathrm{p}=0.034)$ and KI-67 status 
$(p=0.034)$ were significantly associated with the presence of bone metastasis in the subjects of this study. The detailed data of the bivariate analysis is provided in Table 1.

\section{DKK-1 Expression Analysis in Bone Metastasis Breast Cancer}

$\mathrm{H}$-Score was calculated from each sample. The ROC curve is shown in Figure 1. The cut-off point was determined using the graphs of sensitivity and specificity provided in Figure 2. Determined cut off value of $\mathrm{H}$ - Score was 142.5; we considered DKK-1 expression as high if the $\mathrm{H}-$ Score value was $\geq 142.5$ and low if the $\mathrm{H}-$ Score value was < 142.5 .

\section{Association Between Expression of DKK-1 and Bone Metastasis}

We found a statistically significant difference in the mean $\mathrm{H}$-Score values between bone metastasis group (163.5 \pm 24.55$)$ and non-bone metastasis group (123.6 \pm 25.59$)$. The magnitude of difference was 39.9 with $95 \%$ CI of 28.5 - 51.4. Chi Square test indicated significant association between expression of DKK-1 and bone metastasis of breast cancer patients $(\mathrm{p}<0.001)$ as presented in Table 2 . Breast cancer patients with high DKK-1 expression were 12 times more likely to develop bone metastasis compared to those with low expression of DKK- 1 .

Calculation of sensitivity, specificity, positive predictive value, negative predictive value, positive likelihood ratio, negative likelihood ratio, and the

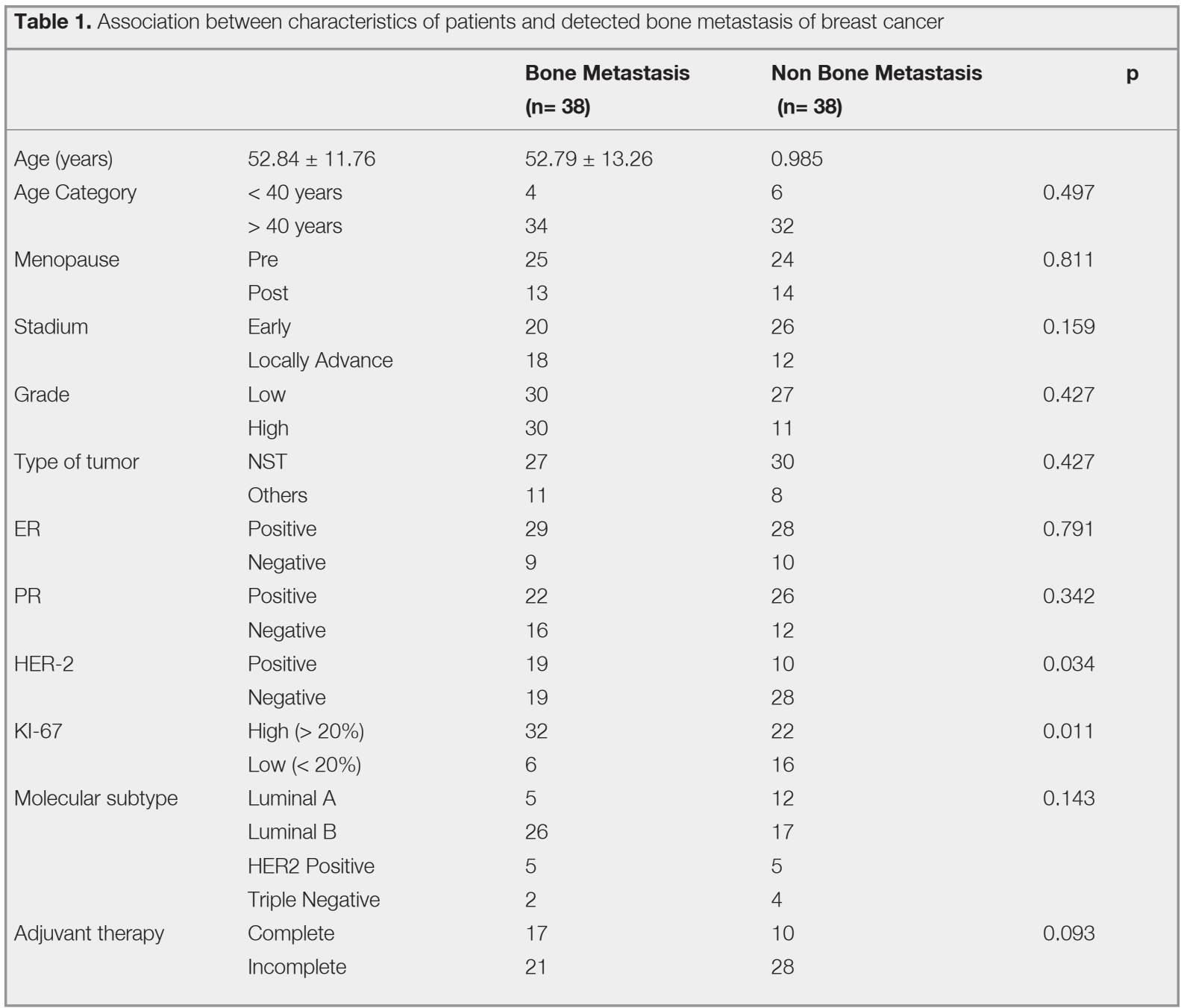




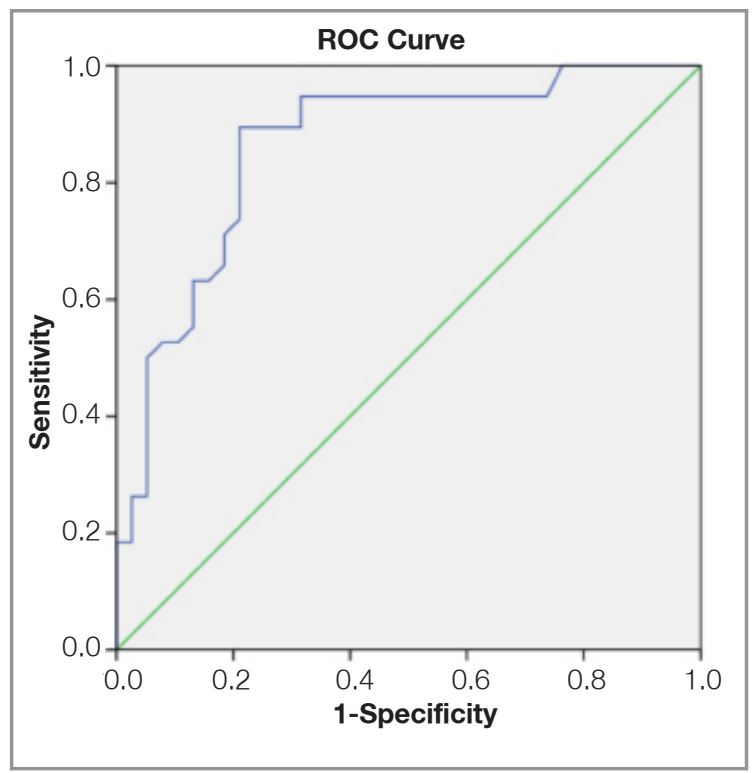

Figure 1. ROC Curve analysis of the DKK-1 biomarker expression

accuracy of DKK-1 expression for predicting bone metastasis in breast cancer patients is provided in Table 3. This calculation is conducted based on the results of this study, shown in Table 2.

\section{Association Between Clinicopathological Fac- tors and DKK-1 Expression}

Bivariate analysis showed no statistically significant association between clinicopathological factors and expression of DKK-1. Multivariate analysis was conducted between clinicopathological factors on the expression of DKK-1 by including factors that had a $p$ value $<0.25$ in the previous bivariate analysis. This included age, menopause status, and HER-2. From this analysis, no factors had significant associations with DKK-1 expression ( $\mathrm{p}>0.05)$. The details on bivariate and multivariate analysis of association between clinicopatho-

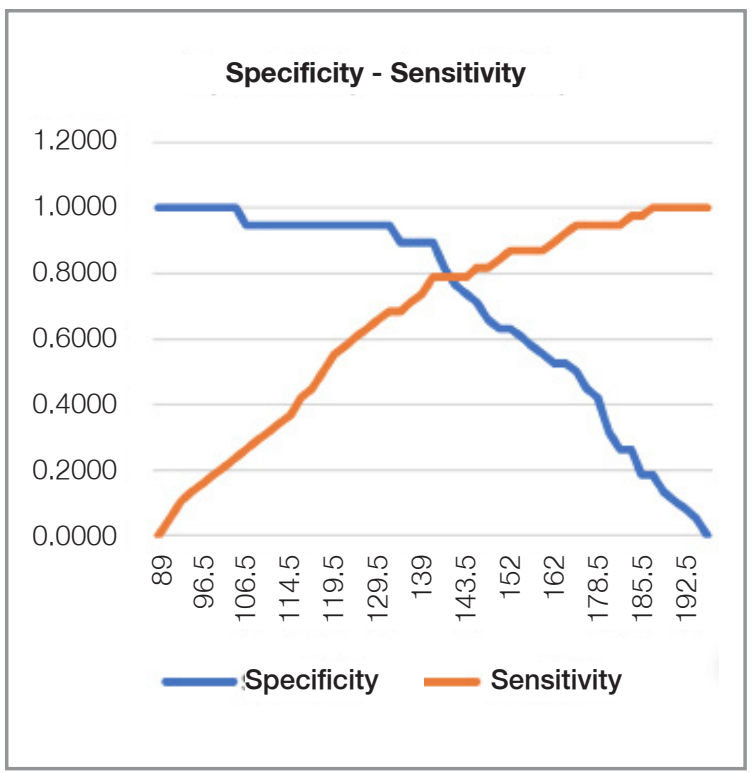

Figure 2. Specificity and sensitivity graphs of DKK-1 biomarker detection. The cut-off point of $\mathrm{H}$ - Score determined was 142.5

logical factors and DKK-1 expression is shown in Table 4.

\section{DISCUSSION}

\section{Patient Characteristics}

The average age of subjects in this study was 52.84 \pm 11.76 years in the bone metastasis group and $52.79 \pm 13.26$ years in the non-bone metastasis group. Purushotam et al. found a reduced risk of distant metastasis with age, but there was an increased risk of death at age $>50$ years. ${ }^{25}$ In this study, there were more premenopausal patients than postmenopausal patients in both groups. Based on a retrospective study in the United Kingdom, postmenopausal women (63\% vs $43 \%, \mathrm{p}=0.0002)$ had a significant single risk of bone metastasis, compared to a combination of bone and visceral me-

Table 2. Relationships between DKK-1 expression and bone metastasis

\begin{tabular}{|c|c|c|c|c|c|}
\hline & & \multicolumn{2}{|c|}{ Bone Metastasis } & \multirow{2}{*}{$\begin{array}{l}\text { OR } \\
(95 \% \mathrm{Cl})\end{array}$} & \multirow[t]{2}{*}{$\mathbf{p}$} \\
\hline & & $(+)$ & $(-)$ & & \\
\hline \multirow[t]{2}{*}{ DKK-1 } & High & 29 & 8 & $12.083(4.101-35.600)$ & $<0.001$ \\
\hline & Low & 9 & 30 & & \\
\hline
\end{tabular}




\begin{tabular}{|ll|}
\hline $\begin{array}{l}\text { Table 3. Indicators on diagnostic test of DKK-1 for bone } \\
\text { metastasis }\end{array}$ & Value \\
\hline Indicators & $76.3 \%$ \\
\hline Sensitivity & $78.9 \%$ \\
Specificity & $78.3 \%$ \\
Positive predictive value & $76.9 \%$ \\
Negative predictive value & 3.616 \\
Positive likelihood ratio & 0.301 \\
Negative likelihood ratio & $77.6 \%$ \\
Accuracy & \\
\hline
\end{tabular}

tastasis. ${ }^{26}$ In both groups patients were found to have more early stages than local advanced stages. Tumor staging is an independent risk factor for tumor metastasis according to various studies. ${ }^{27,28}$ The number of patients with low grade tumors was higher than patients with high grade tumors in both groups of this study. Bone metastasis is more common in low grade breast cancer, but this does not appear to be true in breast cancer luminal subtype A. ${ }^{28-30}$ In terms of tumor types, we observe higher numbers of tumors with NST type compared to other types in both groups. However, histopathological subtypes were not associated with risk of occurrence bone metastasis in a previous study. ${ }^{31}$ In both groups, more subjects had positive hormonal receptor (ER / PR) expression. Lee et al. found that $85 \%$ of single bone metastasis in breast can-

\begin{tabular}{|c|c|c|c|c|c|c|}
\hline \multirow{2}{*}{ Factors } & & \multicolumn{2}{|c|}{ Bivariate analysis } & \multirow[b]{2}{*}{$\bar{p}$} & \multicolumn{2}{|l|}{ Multivariate analysis } \\
\hline & & High & Low & & OR (95\% Cl) & $\mathbf{p}$ \\
\hline \multicolumn{2}{|l|}{ Age (years) } & $54.92 \pm 13.79$ & $50.82 \pm 10,83$ & 0.156 & $0.983(0.911-1.060)$ & 0.654 \\
\hline \multirow[t]{2}{*}{ Age category } & $<40$ years & 4 & 6 & 0.555 & & \\
\hline & $>40$ years & 33 & 33 & & & \\
\hline \multirow[t]{2}{*}{ Menopause } & Pre & 20 & 29 & 0.065 & $3.792(0.518-27.775)$ & 0.190 \\
\hline & Post & 17 & 10 & & & \\
\hline \multirow[t]{2}{*}{ Stadium } & Early & 20 & 26 & 0.261 & & \\
\hline & Locally Advance & 17 & 13 & & & \\
\hline \multirow[t]{2}{*}{ Grade } & Low & 5 & 6 & 0.817 & & \\
\hline & High & 32 & 33 & & & \\
\hline \multirow[t]{2}{*}{ Type of Tumor } & NST & 26 & 31 & 0.354 & & \\
\hline & Others & 11 & 8 & & & \\
\hline \multirow[t]{2}{*}{ ER } & Positive & 29 & 28 & 0.508 & & \\
\hline & Negative & 8 & 11 & & & \\
\hline \multirow[t]{2}{*}{ PR } & Positive & 24 & 24 & 0.764 & & \\
\hline & Negative & 13 & 15 & & & \\
\hline \multirow[t]{2}{*}{ HER-2 } & Positive & 17 & 12 & 0.173 & $0.489(0.185-1.290)$ & 0.148 \\
\hline & Negative & 20 & 27 & & & \\
\hline \multirow[t]{2}{*}{$\mathrm{KI}-67$} & High (> 20\%) & 27 & 27 & 0.719 & & \\
\hline & Low (<20\%) & 10 & 12 & & & \\
\hline \multirow[t]{4}{*}{ Molecular Subtype } & Luminal A & 8 & 9 & 0.377 & & \\
\hline & Luminal B & 22 & 21 & & & \\
\hline & HER2 Positive & 64 & 4 & & & \\
\hline & Triple Negative & 1 & 5 & & & \\
\hline \multirow[t]{2}{*}{ Adjuvant Therapy } & Complete & 15 & 12 & 0.374 & & \\
\hline & Incomplete & 22 & 27 & & & \\
\hline
\end{tabular}


cer with positive hormonal receptors was associated with good clinical outcomes..$^{32}$ Based on the bivariate analysis, HER-2 $(\mathrm{p}=0.034)$ and KI-67 expression $(\mathrm{p}=0.034)$ were significantly associated with the presence of bone metastasis in the subjects of this study. Savci-Heijink et al.'s findings supported that HER-2 amplification lead to a bigger chance of bone metastasis in breast cancer patients. ${ }^{33}$ Moreover, Inari K et al. also described a high KI-67 index in breast cancer patients with brain, visceral and bone metastasis with poor overall survival $(p=0.011) .{ }^{34}$ For the molecular subtype, luminal B subtype was the most commonly found in subjects of this study. However, Wu Q et al. found that almost all molecular subtypes tended to occur in bone metastasis..$^{35}$ In both groups, we observe a larger percentage of subjects who did not complete adjunctive therapy compared to subjects who did. We found the risk of bone recurrence did not change even though the patient had received adequate adjunctive therapy. Pulido et al. found a considerable effect from adjuvant therapy in metastatic patterns. ${ }^{28}$ For example, tamoxifen is known to reduce the risk of bone metastasis in a group of patients with positive ER expression, and this effect continues until after therapy is stopped. But most of these previous studies are also retrospective and the heterogeneity of the findings may result from the variety of treatment regimens used. For adjuvant chemotherapy, the results are still unclear.

In the end, the authors realize the heterogenicities on baseline characteristics of subject as a weakness in this study.

\section{Association Between Expression of DKK-1 and Bone Metastasis}

In the bone metastasis group, we found high expressions of DKK-1 in 29 subjects and low expressions in 9 subjects. There were 8 subjects with high expressions of DKK-1 and 30 subjects with low expression in the non-bone metastatic group. Analysis with Chi square test resulted in an Odd Ratio (95\% CI) of 12.083 (4.101-35.600). Hence, breast cancer patients with high DKK-1 expression are more likely to have bone metastasis. This is supported by a study by Voorzanger-Rousselot $\mathrm{N}$ et al. where expression of DKK-1 was higher in bone metastatic breast cancer patients compared to complete remission breast cancer patients $(p=0.016)$, breast cancer patients without bone metastasis ( $\mathrm{p}<$ $0.0001)$ and healthy women $(\mathrm{p}=0.047){ }^{36}$

In this study, DKK-1 diagnostic test for bone metastasis have a sensitivity value of $76.3 \%$, specificity of $78.9 \%$, positive predictive value of $78.3 \%$, negative predictive value of $76.9 \%$, positive likelihood ratio of 3.616 , negative likelihood ratio of 0.301 , and $77.6 \%$ accuracy. Therefore, detection of DKK-1 expression can be considered to be used both in screening and as a predictor of the presence of bone metastasis in breast cancer patients. Our findings are consistent with the sensitivity and specificity of other biomarkers of bone metastasis. Sufrida et al. found osteonectin expression to have $80 \%$ sensitivity and $83.3 \%$ specificity, while PTHrP expression had a sensitivity of $83.3 \%$ and specificity of $63.3 \% .{ }^{37}$ Ibrahim et al. reported breast cancer with high expression of CXCR4 was associated with bone metastasis with a specificity of $90 \%$, while RANK expression was positively associated with bone metastasis with a specificity of $80 \%$. The sensitivity of ER was $80 \%$, but the specificity was low at $30 \%{ }^{38,39}$ Based on these data, we concluded that expression of DKK-1 can be used as a predictor factor for bone metastasis in breast cancer. Hence, administration of bisphosphonate therapy can be given to patients with breast cancer with high expression of DKK-1, both as a therapeutic and preventive therapy.

\section{Association Between Clinicopathological Factors and DKK-1 Expression}

Bivariate analysis showed no statistically significant associations between clinicopathological factors and expression of DKK-1. Moreover, based on multivariate analysis, no factors had significant associations with DKK-1 ( $>$ > 0.05). Based on these results, it can be concluded that DKK-1 is an independent factor in the occurrence of bone metastasis in breast cancer. Our finding is supported by Suzuki et al.'s study who also found that there were no significant associations $(p>0.05)$ between clinicopathological factors and the expression of DKK $-1 .{ }^{40}$ In contrast, a study conducted by Zhou 
SJ et al, reported that stage, grade, lymph node metastasis and HER-2 expression were associated significantly with DKK-1 expression $(\mathrm{p}<0.05) .{ }^{41}$

In conclusion, DKK-1 expression can be a predictor of the incidence of bone metastasis in breast cancer. Despite the low accuracy of this predictor, this study may broaden clinicians knowledge on DKK-1 expression test usage on breast cancer patients compared to other modalities. No clinicopathological factors are significantly associated with the expression of DKK-1 in breast cancer patients with bone metastasis.

\section{Acknowledgment}

The authors would like to show gratitude to $d r$. Kevin Varian for assisting the publication of this study.

\section{REFERENCES}

1. Jemal A, Bray F, Center MM, et al. Global cancer statistics. CA Cancer J Clin 61: 69-90, 2011.

2. Youlden DR, Cramb SM, Yip CH, Baade PD. Incidence and Mortality of Female Breast Cancer in the Asia-Pacific Region. Cancer Biol Med 11: 101-115, 2014.

3. Cancer Prevention National Committee. Guidelines on Breast Cancer Management. Jakarta: Ministry of Health Republic Indonesia, 2015: 1.

4. Kuchuk I, Hutton B, Moretto P, et al. Incidence, consequences and treatment of bone metastases in breast cancer patients-experience from a single cancer centre. J Bone Oncol 2: 137-144, 2013.

5. Coleman RE. Metastatic bone disease: Clinical features, pathophysiology and treatment strategies. Cancer Treat Rev 27: 165-176, 2001.

6. Fang J, Xu Q. Differences of osteoblastic bone metastases and osteolytic bone metastases in clinical features and molecular characteristics. Clin Transl Oncol 17: 173-179, 2015.

7. Harries M, Taylor A, Holmberg L, et al. Incidence of bone metastases and survival after a diagnosis of bone metastases in breast cancer patients. Cancer Epidemiol 38: 427-434, 2014.

8. D'Oronzo S, Brown J, Coleman R. The value of biomarkers in bone metastasis. Eur J Cancer Care (Engl) 26: 6, 2017.

9. Bu G, Lu W, Liu CC, et al. Breast cancer-derived Dickkopf1 inhibits osteoblast differentiation and osteoprotegerin expression: Implication for breast cancer osteolytic bone metastases. Int J Cancer 123: 1034-1042, 2008.
10. Baron R, Kneissel M. WNT signaling in bone homeostasis and disease: from human mutations to treatments. Nat Med 19: 179-192, 2013.

11. Mariz K, Ingolf JB, Daniel H, et al. The Wnt inhibitor dickkopf-1: a link between breast cancer and bone metastases. Clin Exp Metastasis 32: 857-866, 2015.

12. Mao B, Wu W, Davidson G, et al. Kremen proteins are Dickkopf receptors that regulate Wnt/ $\beta$-catenin signalling. Nature 417: 664-667, 2002.

13. Gregory CA, Singh H, Perry AS, Prockop DJ. The Wnt signaling inhibitor dickkopf-1 is required for reentry into the cell cycle of human adult stem cells from bone marrow. J Biol Chem 278: 28067-28078, 2003.

14. Mikheev AM, Mikheeva SA, Liu B, et al. A functional genomics approach for the identification of putative tumor suppressor genes: Dickkopf-1 as suppressor of HeLa cell transformation. Carcinogenesis 25: 47-59, 2004.

15. You L, He B, Uematsu K, et al. Inhibition of Wnt-1 signaling induces apoptosis in beta-catenin-deficient mesothelioma cells. Cancer Res 64: 3474-3478, 2004.

16. Loffredo FS, Pancoast JR, Cai L, et al. Targeted delivery to cartilage is critical for in vivo efficacy of insulin-like growth factor 1 in a rat model of osteoarthritis. Arthritis Rheumatol (Hoboken, NJ) 66: 1247-1255, 2014.

17. Jiang $T$, Huang $L$, Zhang S. DKK-1 in serum as a clinical and prognostic factor in patients with cervical cancer. Int J Biol Markers 28: 221-225, 2013.

18. Darlavoix $T$, Seelentag W, Yan P, et al. Altered expression of CD44 and DKK1 in the progression of Barrett's esophagus to esophageal adenocarcinoma. Virchows Arch 454: 629-637, 2009.

19. Wirths O, Waha A, Weggen S, et al. Overexpression of human Dickkopf-1, an antagonist of wingless/WNT signaling, in human hepatoblastomas and Wilms' tumors. Lab Investig 83: 429-434, 2003.

20. Gomceli I, Bostanci EB, Ozer I, et al. A novel screening biomarker in gastric cancer: Serum Dickkopf-1. Hepatogastroenterology 59: 1661-1664, 2012.

21. Feldmann R, Schierl M, Fink AM, et al. Serum levels of glycoprotein Dickkopf-1 in patients with cutaneous malignant melanoma: a prospective pilot study. Dermatology 222: 171 175, 2011.

22. González-Sancho JM, Aguilera O, García JM, et al. The Wnt antagonist DICKKOPF-1 gene is a downstream target of $\beta$-catenin/TCF and is downregulated in human colon cancer. Oncogene 24: 1098-1103, 2005.

23. Xu WH, Liu Z Bin, Yang C, et al. Expression of Dickkopf-1 and beta-catenin related to the prognosis of breast cancer patients with triple negative phenotype. PLoS One 7(5): 1-7, 2012.

24. Hong SA, Yoo SH, Lee HH, et al. Prognostic value of Dickkopf-1 and B-catenin expression in advanced gastric cancer. BMC Cancer 18-506, 2018. 
25. Purushotham A, Shamil E, Cariati M, et al. Age at diagnosis and distant metastasis in breast cancer - a surprising inverse relationship. Eur J Cancer 50: 1697-705, 2014.

26. Coleman R, Smith P, Ruben R. Clinical course and prognostic factors following bone reccurence from breast cancer. $\mathrm{Br}$ J Cancer 77: 336-340, 1998.

27. Bertozzi S, Londero A, Cedolini C, et al. Prevalence, risk factors, and prognosis of peritoneal metastasis from breast cancer. Springerplus 4: 688, 2015.

28. Pulido C, Vendrell I, Ferreira AR, et al. Bone metastasis risk factors in breast cancer. Ecancermedicalscience 11: 715, 2017.

29. Chen J, Zhu S, Xie X, et al. Analysis of clinicopathological factors associated with bone metastasis in breast cancer. J Huazong Univ Sei Technolog Med Sci 33: 122-125, 2013.

30. James J, Evans A, Pinder S, et al. Bone metastasis from breast carcinoma: histopathological - radiological correlations and prognostic features. $\mathrm{Br} J$ Cancer 89: 660-665, 2003.

31. Diessner J, Wischnewsky M, Stuber T, et al. Evaluation of clinical parameters influencing the development of bone metastasis in breast cancer. BMC Cancer 16: 307, 2016.

32. Lee S, Park S, Ahn H, et al. Implications of bone-only metastasis in breast cancer: Favorable preferences with excellent outcomes of hormone receptor positive breast cancer. Cancer Res Treat 43: 89-95, 2011.

33. Savci-Heijink C, Halfwerk H, Hooijer G, et al. Retrospective analysis of metastatic behaviour of breast cancer subtipes. Breast Cancer Res Treat 150: 547-557, 2015.

34. Inari $\mathrm{H}$, Suganuma N, Kawachi K, et al. Clinicopathological and prognostic significance of $\mathrm{KI}-67$ immunohistochemical expression of distant metastatic lesions in patients with metastatic breast cancer. Breast Cancer 24: 748-755, 2017.

35. Wu Q, Li J, Zhu S, et al. Breast cancer subtipes predict the preferential site of distant metastates: a SEER based study. Oncotarget 8: 27990-27996, 2017.

36. Voorzanger-Rousselot N, Goehrig D, Journe F, et al. Increased Dickkopf-1 expression in breast cancer bone metastases. Br J Cancer 97: 964-970, 2007.

37. Sufrida N, Hernowo B, Suryanti S. Parathyroid hormonerelated protein (PTHrP) dan osteonectin (OSN) sebagai petunjuk adanya penentu metastasis ke tulang pada karsinoma payudara duktal invasif. Indonesia J Pathol 20: 15-24, 2011.

38. Ibrahim T, Sacana E, Gaudio M, et al. Role of RANK, OPG and CXCR4 Tissue Markers in Predicting Bone Metastases. Clin Breast Cancer 11: 369-375, 2011.

39. Ibrahim T, Sacana E, Gaudio M, et al. Accuracy of RANK OPG and CXCR4 compared to hormone receptors in predicting bone metastases in patients with breast cancer. $J$ Clin Oncol 28: 11507, 2010.
40. Suzuki $H$, Toyota $M$, Caraway $H$, et al. Frequent epigenetic inactivation of Wnt antagonist genes in breast cancer. $\mathrm{Br} J$ Cancer 98: 1147-1156, 2008.

41. Zhou SJ, Zhuo SR, Yang XQ, et al. Serum Dickkopf-1 expression level positively correlates with a poor prognosis in breast cancer. Diagnostic Pathology 9: 161, 2014.

\section{Correspondence:}

Erwin Danil YULIAN, M.D., Ph.D.

Division of Surgical Oncology, Department of Surgery

Dr. Cipto Mangunkusumo General Hospital

Faculty of Medicine Universitas Indonesia

Jakarta / INDONESIA

Tel: $\quad+6281315249627$

e-mail: erwin.yulian@ui.ac.id

\section{ORCIDs:}

Faruly Wijaya S. Limba

0000-0003-4274-2179

Erwin Danil Yulian

Diani Kartini

Bob Andinata

Hartono Tjahjadi

Dhanasari Vidiawati
0000-0003-4149-4281

0000-0001-8686-2987

0000-0002-0607-9747

0000-0001-5725-371X

0000-0001-7133-5009 